\title{
Verkörperung: Bild und Experiment bei Edgar Wind und die aktuelle Lage der morphologischen Forschung
}

\begin{abstract}
Edgar Wind's The Experiment and Metaphysics examines, in the sense of Goethe's morphological project, the common structures of scientific and artistic thinking in the light of the reflective value of the image and the technological processes of research and construction of reality. In Wind's philosophy of embodiment the image plays a role in the artistic field comparable to the function of experiments in science. The picture is neither a 'copy' nor just a 'part' of reality, but rather an 'embodiment': at the same time the 'result' of human activity and a 'condition' for all interpretation of the world. It opens up as an embodiment to a temporality which is not just linear but 'configured', i.e. morphologically constituted. The aim of this chapter is to reconstruct the main articulations of the wind proposal, while testing the possibility of linking image and technology experiments through the interactions of current augmented reality.
\end{abstract}

Edgar Winds Hauptwerk Das Experiment und die Metaphysik entwickelt eine Reflexion die, im Sinne von Goethes morphologischem Vorhaben, die gemeinsamen Strukturen von wissenschaftlichem und künstlerischem Denken im Licht des reflektierenden Wertes des Bildes und der technologischen Verfahren der Erforschung und Konstruktion des Realen untersucht.

Winds Philosophie der Verkörperung bietet eine breite Basis für die Reflexion über die Beziehung zwischen Wissenschaft und kulturgeschichtlicher Methode, Technik und ästhetischem Denken. Nach dem von Wind unterstützten Konzept spielt das Bild im künstlerischen Bereich eine Rolle, die vergleichbar ist mit der Funktion, die das Experiment in der Wissenschaft spielt; der Wert des theoretischen Bezugsrahmens und die Realität der kognitiven und technologischen Werkzeuge werden daher in der Konstruktion des Bildes und in der methodologischen Reflexion, die darauf ausgeübt wird, auf die Probe gestellt.

Das Bild ist weder ,Kopie“ noch bloß ,Teil‘ der Wirklichkeit, sondern vielmehr ,Verkörperung': gleichzeitig ,Resultat‘ des menschlichen Handelns bzw. Herstellens und ,Bedingung، aller Deutung und Auseinandersetzung mit der Welt. Auf diese Weise öffnet sich das Bild als eine Verkörperung zu einer dem Bild eigenen Zeitlichkeit, nicht bloß linear, sondern ,konfiguriert‘, also genau morphologisch konstituiert.

Ә OpenAccess. (C) 2021 Salvatore Tedesco, publiziert von De Gruyter. (cc))BY-NC-ND Dieses Werk ist lizenziert unter einer Creative Commons Namensnennung - Nicht kommerziell - Keine Bearbeitung 4.0 International Lizenz. https://doi.org/10.1515/9783110674194-013 
Der vorliegende Beitrag zielt darauf ab, eine Rekonstruktion der wichtigsten Artikulationen des Wind-Vorschlags zu liefern, wobei gleichzeitig die Relevanz und die Möglichkeit der Verknüpfung von Bild und Technologieexperimenten durch die Interaktionen der aktuellen Augmented Reality auf die Probe gestellt werden.

In seinem kurzen, 1913 verfassten Vortrag über Luftschiff und Tauchboot in der mittelalterlichen Vorstellungswelt, erzählt Aby Warburg von zwei flämischen Wandteppichen aus dem fünfzehnten Jahrhundert (damals im Palazzo DoriaPamphili in Rom aufbewahrt und nun in die Villa Doria in Genua verlegt), wo einige märchenhafte Episoden der Geschichte von Alexander dem Großen erzählt werden, die hauptsächlich aus dem Roman d'Alexandre in der Fassung von Jean Wauquelin stammen.

Im zweiten der beiden Wandteppiche - der die reifen Taten des großen Makedons erzählt - sehen wir den König, „wie er in einem von vier Greifen gezogenen Metallgehäuse gen Himmel fährt“, und gleich daneben „wie er in einem gläsernen Fasse ins Meer hinabgelassen wird“ (Warburg 1932, 243).

Warburg beschreibt den Weg des großen Alexander, einen Weg, der ihn an die Enden der Erde drängt und ihn dazu bringt, mit der Kraft der Elemente zu konkurrieren. Wenn wir über dem einzigartigen Luftschiff Alexanders sehen, daß Gott der Vater von Engeln umgeben ist, die den Weg für einen weiteren Aufstieg sperren, erinnern wir uns daran, „daß es dem Sterblichen versagt sei, über die vierte Region des Feuers bis in den Himmel vorzudringen“ (Warburg 1932, 245).

Nicht weit entfernt sehen wir aber „Alexander im gläsernen Fass, in jeder Hand eine brennende Fackel“ (Warburg 1932, 246), der in die Tiefe des Meeres abtaucht, während seine Soldaten jetzt mit ihren Bombarden das Feuer entscheidend beherrschen (,ein Kanonier feuert gerade seine Bombarde ab, sein Gesicht mit der anderen Hand vor den entflammten Pulvergasen schützend“, Warburg 1932, 247). In den Geistern dieser mittelalterlichen Phantasie erkennt Warburg jedoch die Spüren alter Sonnenkulte, die mit dem Osten des römischen Reiches verbunden sind, und damit die Entstehung einer fast „ungewollten Symbolik“, in der die mittelalterliche und die moderne Welt „die Gegensätzlichkeit ihres seelischen Aufbaues“ (Warburg 1932, 247) messen.

Der Aufstieg Alexanders des Großen, der in den Gesichtszügen des Herzogs Karl von Burgund identifiziert wurde, wird so zum Ausdruck des Willens, „sich antiker Größe zu erinnern“ (Warburg 1932, 249), und es ist ein Wille, der sich trotz aller Unterschiede, die „der überladene Trachtenrealismus und die romantische Märchenphantastik“ impliziert, im Fall des flämischen Wandteppich „mit derselben inneren Energie auftritt wie in Italien“, in der Heimat der Renaissance des Klassizismus; und eben deshalb, fügt Warburg hinzu, „diese ,burgundische Antike‘ ebenso wie die, italienische‘ ihren wesentlichen und eigenartigen Anteil hat 
an der Erzeugung des modernen, auf die Beherrschung der Welt gerichteten Menschen. Noch scheint ihm die Feuerregion (...) unnahbar, während er doch schon das Flammenelement in seinen Feuerschlünden gebändigt und dienstbar unter den Händen hält“ (Warburg 1932, 249).

Ich gehe gerne von diesem Warburg'schen Bild aus, weil es uns in seiner scheinbaren Einzigartigkeit und sogar Extravaganz auf die direkteste Weise zu der Frage führt, mit der ich mich gerne beschäftigen würde, und die kurz als Relevanz der Technik im heutigen morphologischen Denken formuliert werden kann.

Ich beziehe mich auf eine doppelte Relevanz in Bezug auf die Definition desselben morphologischen Projekts, das als Reflexion über die Natur des Wissens und die Konfiguration des Wissens sowie über die menschliche Natur und die Art und Weise, in der eben diese menschliche Natur von der Frage der Technik durchquert wird, beabsichtigt ist.

Man könnte vermuten es sei darin eine Art Ungenauigkeit mit Bezug auf Goethes Ansatz einer Morphologie, da Goethe ohne weiteres alle ,Anthropologisierung‘ des Gestalt-Begriffs widerlegte. Ich möchte jedoch feststellen, daß der problematische Aufbau, von dem wir sprechen, weitaus komplexer ist, als eine bloße Anthropologisierung der Gestalt vermuten lässt: der Verweis auf die Technik verschiebt die Aufmerksamkeitsachse vom ,menschlichen Subjekt` zur ,Umweltdynamik‘ der Lebensform, von der dieses Subjekt Ausdruck ist.

Der Ausgangspunkt dieser kurzen Überlegungen betrifft unsere eigene Zeit, d. h. die Frage nach der Augmented Reality und die Form des Wissens in einem Kontext, der durch die wirbelnde Zirkulation von Bildern gekennzeichnet ist. Es handelt sich von Bildern, die sich als Stationen und Indizien aufstellen, die für ein öffentliches Teilen wichtig sind, die aber nichtsdestoweniger nur sehr schwierig einen symbolischen Wert erwerben und verkörpern. Um zu einer etwas reflektierenden Sichtweise über diesen Zustand $\mathrm{zu}$ gelangen, halte ich es für sinnvoll, einen Schritt zurück zu gehen und die Analyse auf einen der wichtigsten Momente des morphologischen Denkens des 20. Jahrhunderts zu stützen, der durch die Reflexion von Edgar Wind in seiner reifsten Phase dargestellt wird. Eine Reflexion, im Kontext derselben Warburg-Schule geboren, die leider - wie bekannt - durch den Nationalsozialismus und die Flucht von Edgar Wind in England und dann in den Vereinigten Staaten gewaltsam unterbrochen wurde.

Ich beziehe mich speziell auf das Konzept der ,Verkörperung' des technischen Experiments, von Wind im Meisterwerk Das Experiment und die Metaphysik von 1934 vorgeschlagen; ein Konzept, das zur Idee einer radikalen Beteiligung der Technik an der Bestimmung der menschlichen Natur führt.

Winds Philosophie der Verkörperung bietet eine breite Basis für die Reflexion über die Beziehung zwischen Wissenschaft und kulturgeschichtlicher Methode, Technik und ästhetischem Denken. Nach dem von Wind unterstützten Konzept 
spielt das Bild im künstlerischen Bereich eine Rolle, die vergleichbar ist mit der Funktion, die das Experiment in der Wissenschaft spielt; der Wert des theoretischen Bezugsrahmens und die Realität der kognitiven und technologischen Werkzeuge werden daher in der Konstruktion des Bildes und in der methodologischen Reflexion, die darauf ausgeübt wird, auf die Probe gestellt.

Das Bild ist weder ,Kopie“ noch bloß ,Teil‘ der Wirklichkeit, sondern vielmehr ,Verkörperung‘: gleichzeitig ,Resultat‘ des menschlichen Handelns bzw. Herstellens und ,Bedingung، aller Deutung und Auseinandersetzung mit der Welt. Auf diese Weise öffnet sich das Bild als eine Verkörperung zu einer dem Bild eigenen Zeitlichkeit, nicht bloß linear, sondern ,konfiguriert‘, also genau morphologisch konstituiert.

Ich werde mich hier nicht einer analytischen Rekonstruktion des argumentativen Kontextes von Winds Thesen widmen; mein Ziel ist es vielmehr, sozusagen die ,Systemeffekte' hervorzuheben, die diese Thesen auf unser Verständnis von Bildern und Lebensformen selbst erzeugen.

Die Einstellung eines technischen Instruments, mit dem eine bestimmte theoretische Annahme in einer bestimmten Situation erprobt werden soll, ist nach Wind weder eine reine Sache des (logischen) Denkens, noch ist sie bloß empirisch motivierbar; vielmehr kann sie „nur als ein Akt symbolischer Repräsentation definiert werden“ (Wind 2001, 75).

Da das technische Instrument als Element der physischen Welt den gleichen Gesetzen unterworfen ist, deren Erprobung das Instrument andererseits beitragen soll, wird eine solche Konditionierung als Ursprung der Forschung dienen; die Wahl des Instruments ,verkörpert‘ ein gesamtes ,System der Welt‘, und dies - nach einer Art paradoxer Umkehrung des Kantismus - nur aus der Begrenzung unseres Horizonts möglich ist.

Für die Konstruktion des physischen Instruments und für die Interpretation der historischen und künstlerischen Tatsache, sagt Wind, brauchen wir „ein Urteil über die (...) Gesamtkonstellation, an der sie teilhaben“; jedesmal - bei der Durchführung der experimentellen Forschung ebenso wie beim Aufbau der ästhetischen Erfahrung - herstellen wir „diese Beziehung vom Teil zum Ganzen“ (Wind 2001, 84-85).

Unter diesen Bedingungen erhebt sich das Bild zur Rolle eines ,Aktes der symbolischen Repräsentation'. All dies hat offensichtlich wenig mit der ,Verbreitung' und dem ,Austausch'von Bildern zu tun, aber viel mit der Überprüfung der Bedingungen, unter denen das Bild selbst auf das Ganze verweist, und mit der Art und Weise, wie das Bild seine Umwelt bewohnt. Deshalb konfiguriert die Interaktion mit dem technologischen Instrument das, was Wind als „ein metaphysisches Signal“ (Wind 2001, 87) definiert. 
Das physikalische Experiment und die ästhetisch-künstlerische Erfahrung eröffnen damit einen metaphysischen Hintergrund: die methodologische Anlage, in der das Bild konfiguriert ist, beweist seine Gültigkeit darin, daß sie (wie Wind es ausdrückt) eine bestimmte theoretische Hypothese erprobt, indem sie „einen völlig meta-logischen und daher auch meta-physischen Akt provoziert“ (Wind 2001, 219).

Wind definiert ,Akt der Verkörperung` die plötzliche Erzeugung einer solchen wechselseitigen Konfrontation zwischen einem konditionierten Element und der Gesamtheit des realen Systems, bei der sich wechselseitig Instrument und System, Element und Ganzheit an- und durcheinander bewähren. Er spricht auch von experimentum crucis; mit diesem Ausdruck meint er ein Experiment, bei dem Symbole, indem sie ,verkörpert` werden, ihre Realität auf beobachtbare Weise manifestieren können.

Das ist genau das, was bei der Gestaltung des ästhetisch auffallenden Bildes geschieht, dessen metaphysische Substanz dem der neokantianischen Tradition so nahe liegenden Kreislauf zwischen Kunst-Theorie und -Geschichte entgeht: eben im einzigen Verkörperungsakt, mittels seiner historischen Lage und seiner bildlich-medialen und relationalen Bestimmtheit, werden die systematischen Annahmen verwirklicht und auf die Probe gestellt.

Mit dem Ausdruck ,metaphysischer Akt‘ will Wind einerseits die Auswirkungen des Gebrauchs technischer Instrumente auf die Konstitution der Realität hervorheben, andererseits die Art und Weise, wie die menschliche Lebensform in der konkreten Tatsache der Erfahrung (in ihrer letztlich unvermeidlichen technischen Vermittlung betrachtet) kraftvoll provoziert und in Frage gestellt wird.

Nach Wind geht die Reflexion über die menschliche Natur also von der instrumentellen Vermittlung des Wissens aus, das heißt von seiner Verkörperung in einem Bild, in dem sich Element und Totalität gegenüberstehen: ein unendlicher Geist, beobachtet Wind, kennt keine Metaphysik in dem Sinne, daß es für ihn keine Gegenstände gibt, die über unmittelbare Einsicht hinausgehen; dabei kennt er jedoch nicht einmal die Empirie, also gerade die instrumentelle Vermittlung von Wissen, seine ,Verkörperung‘ als bildnerische Umsetzung.

„Ein endlicher Geist aber“, setzt Wind fort, ,ist an den Gebrauch von Instrumenten gebunden. Denn er weiß sich selbst und die Werkzeuge seiner Erkenntnis als Teile der Welt, die er zu erkennen bestrebt ist, und muß daher das ,Weltenstück‘ als Organ verwenden, um das Ganze der Welt zu erfassen“ (Wind 2001, 109).

Aus heutiger Sicht, sorgt eben diese ,symbolische Verbindung' der Technologie mit dem Anspruch an die Weltganzheit für eine Auffassung der Bilder, die eine äußerst innovative Einsicht - so zu sagen - in ihre ,Tätigkeit` fördert. 
Einer solchen „bildaktiven Phänomenologie“ - um es mit Horst Bredekamp (Bredekamp 2010, 22) zu sagen - erarbeitet Wind rechtzeitig einige wichtige Elemente, sich auf der polaren Beziehung zwischen Metaphysik und Empirie basierend. Die ,unlösliche Verbindung' der beiden Begriffe, die gerade in der Verkörperungsakte der Umsetzung des technologischen Prozesses verwirklicht wird, bezieht die einzige Wahl - die einzige Formulierung einer Gestaltung - mit der Formulierung einer Hypothese über das Ganze. Bedingung der Existenz des endlichen Geistes ist seine „Heteronomie“, sagt Wind (Wind 2001, 110): eben diese Tatsache impliziert die metaphysische Relevanz des Experiments, und vielleicht noch mehr impliziert es eine enge Korrelation zwischen natürlichem und künstlichem Wesen, die sozusagen eine irreversible Veränderung des endlichen Wesens hervorruft. Es handelt sich einer „Selbstverwandlung des Menschen“ (Wind 2001, 267), wie es Wind bald definieren wird.

Die technische Vermittlung von Erfahrung erscheint bei Wind daher nicht nur unvermeidbar, sondern Bedingung derselben Geschichtlichkeit der menschlichen Erfahrung und des Aufbaus einer Erinnerung an das eigene Werden.

„'Erfahrung - schreibt Wind - „ist für einen Gott ein sinnloser Begriff; für ein Tier (...) ist es ein biologischer Prozeß der Gewöhnung. Für den Menschen aber ist Erfahrung Erprobung durch Verwirklichung, d.h. Geschichte. Daher gibt es nur für ihn - im strengen Sinn des Wortes - ,Erinnerung،“ (Wind 2001, 112). Diese technische Prägung und Gestaltung der Erfahrung - füge ich hinzu - ist in unsere Erinnerung eingeschrieben.

Kurz gesagt, der Akzent der Reflexion Winds fällt nicht auf die psychologische Begabung eines Subjekts, sondern auf die symbolischen Implikationen der Verbindung von Erfahrung und Technik: in der Symbolik wird ein gewisser historisch-kultureller Kontext bereits durch die Herstellung von Erinnerung, Erfahrung und Wissen geschaffen. Dies geschieht in einem Prozess, der sowohl kognitive als auch ethische Bedeutung hat.

Wie wir sehen, werden in dieser Betrachtung des symbolischen Umfangs der technisch ausgerüsteten Erfahrung genau die Bedingungen diskutiert, die unser Wissen über die Welt und die Wechselwirkung zwischen der menschlichen Lebensform und ihrer natürlichen und kulturellen Umwelt bestimmen. Wind distanziert sich sowohl von einer historischen Lektüre dieser a priori-Bedingungen (die im deutschen Denken der frühen Jahrzehnte des 20. Jahrhunderts vorherrschend war), als auch von einer (neo-kantianischen) Lektüre, die dazu neigte, sie in eine universelle und unveränderliche Form zu übersetzen. Wind argumentiert vielmehr mit dem Pragmatismus von Clarence Irving Lewis (Lewis 1970). Unsere begrifflichen Schemata informieren die Realität, ändern sich jedoch im Verlauf der Erfahrung, wenn die sich erweiternden Grenzen der Erfahrung ihre (d.h. der 
obengenannten begrifflichen Schemata) Unzulänglichkeit als intellektuelle Instrumente offenbaren.

Nicht weniger bedeutsam ist die Überarbeitung der Frage der historischen Zeit in der Entwicklung der windianischen Reflexion von Erfahrung, Technik und Gedächtnis. Dies ist ein grundlegender Punkt für die Entwicklung der symbolischen Bedeutung der künstlerischen Form, die nicht mehr wie im neo-kantianischen Denken durch ein System fundamentaler Begriffe, die als solche ,durch die Erfahrung unerreichbar‘ bleiben, im Rahmen einer ,Kunstgeschichte“ gesichert sein kann. Entwicklung und Bedeutung der Formen werden nun ganz im Gegenteil beim Herstellen der einzelnen Erfahrung in Frage gestellt (Wind spricht eben in diesem Sinne von experimentum crucis).

Der tradierten Auffassung einer ,linearen Zeit‘ (der Zeit der Chronologie) stellt Wind einen ,konfiguralen‘ Zeitablauf gegenüber. Im Verlauf derselben Erfahrung gerechtfertigt, scheinen die von Zeit zu Zeit auftretenden Konfigurationen spezielle Lösungen zu bilden, die einem Gleichgewicht zwischen spezifischen technischen Problemen und der Formulierung von Bildern der gesuchten Gesamtheit entsprechen. Die ,Emergenz' neuer konfiguralen Zeitbilder markiert - schreibt Wind - das „Auftauchen von Gestalten einer neuen Ordnung aus der ,zufälligen“ Konfiguration der Elemente der alten“ (Wind 2001, 190).

Noch in den Notizen seiner letzten Klassen des Hamburger Unterrichts, die am Vorabend der Flucht von Nazi-Deutschland abgehalten wurden, wird Wind von einer „Rhythmik der geschichtlichen Entwicklung“ sprechen die, die „Regresse der Geschichte“ in einem „gesetzmäßigen Prozeß“ (Wind 2001, 300) nicht ausschließend, eine bestimmte ,Polarität der Symbole‘ absichtlich thematisieren wird.

Der Sinn des geschichtlichen Werdens bietet sich nicht ein für alle Mal als garantierter Besitz an, sondern als eine immer wieder erneuerte ethische Aufgabe in jenen Reflexionspausen, die sich als reale Sammelpunkte des Sinnes erweisen, da sie die Spannungen manifestieren, aus denen die historische Veränderung entstehen kann.

Der Diskontinuität des Entwicklungsgesetzes historischer Prozesse entspricht in Winds Denken eine ebenso tiefgreifende Arbeit in Bezug auf diese ,Sinnregion', die Region sozusagen des Gesamtsinnes, der in der Kunst so wie in der Geschichte den unwiderruflichen Brüchen unterliegt, die durch Veränderungen hervorgerufen werden; ein Sinn, der sich somit an sich unwiderruflich endlich und kontingent offenbart.

Wind schreibt: „Wenn ein Mensch sein Leben vollendet hat, suchen wir den Sinn dieses Lebens zu deuten. Dabei bilden gerade diejenigen Elemente, die den Menschen zu einem endlichen, scheinbar zufälligen Wesen machen, den eigentlichen Gegenstand der Deutung“ (Wind 2001, 209). 
Eine Interpretation, nach der paradoxerweise gerade das Maß der Kontingenz dem Menschen die Bedeutungsregion öffnet: „Indem wir ein Maß der Kontingenz im Kosmos zu entdecken glauben - lesen wir weiter, was Wind schreibt - gewinnt dieser Kosmos einen Sinn für uns. Er enthüllt eine Art pragmatischer Bedeutung“ (Wind 2001, 209).

„Der Mensch eingebunden in die Gesetze der Natur“ (Wind 2001, 297) formt ,für sich' eine immer partielle und begrenzte Bedeutungsebene von den Ereignissen; es handelt sich - um es hier mit einem Wort von Robert Musil zu sagen um ,Teillösungen', die eben im Gleichgewicht von Element und Ganzheit, Natürlichen und Künstlichen sich ergeben.

Auf immer radikalere Weise, so Wind, werden wir mit „technischen Neuerungen“ konfrontiert, „die unsere Umwelt und uns selbst verändern“ (Wind 2001, 268). Die Region des Symbols ist genau dieser Bereich kontinuierlicher Offenheit und Signifikanzrisiko, in dem das Bild in einer ständigen Rückkopplung zwischen technischer Vermittlung und Umgestaltung der Umgebung konfiguriert wird.

Ich fahre schnell fort, um in aller Kürze aus diesen skizzenhaften Analysen einige Schlüsse zu ziehen.

Die Verkörperung der erwähnten Beziehungen findet heute in den sogenannten ,immersiven' Technologien und in der augmented reality eine prominente Betonung. Jedoch nur für eine Untersuchung, die in solchen Verfahren die Verkörperung eines symbolischen Ganzen sieht, bietet die Ausarbeitung von Algorithmen, die die Wiedergabe von Bildern sehr detailliert gestalten und die Wechselwirkungen zwischen natürlichen und künstlichen Elementen perfektionieren können, eine ausreichende Operationsgrundlage.

In keiner Weise geht es darum, eine philosophische Struktur zu überlagern, die sich außerhalb eines konkreten technologischen Prozesses befindet. Ganz im Gegenteil, es sind die konkreten und spezifischen Probleme der Wahrnehmungsund Umweltleistung von virtuellen Produkten, die ein System symbolischer Bedeutungen in Frage stellen, das für die Strukturierung, Planung und Design der einzelnen virtuellen Umwelten wesentlich und relevant ist.

Ganz im Einklang mit den Annahmen von Edgar Wind, ist hier festzustellen, daß jede Neuerung ,am Gegenstand der Forschung auf die Konstruktion des Instrumentariums zurückwirkt: wie ja auch jede Veränderung des Instrumentariums neue Entdeckungsgebiete erschließt“ (Wind 2001, 266).

Die Möglichkeit, einzelne virtuelle Umgebungen $\mathrm{zu}$ bewohnen und $\mathrm{zu}$ durchqueren (letzten Endes: die Möglichkeit der Erfahrung mit den virtual environments), setzt die Lösung spezifischer technischer Anforderungen voraus, gerade weil sie auch ebenso spezifische symbolische Universen verkörpern. 


\section{Literaturverzeichnis}

Bredekamp, Horst (2010): Theorie des Bilsakts, Frankfurt am Main: Suhrkamp Lewis, Clarence Irving (1970): Collected Papers. Stanford: Stanford U.P.

Warburg, Aby (1932): „Luftschiff und Tauchboot in der mittelalterlichen Vorstellungswelt“. In: Aby, Warburg: Gesammelte Schriften, Bd. 1. Hg. von Gertrud Bing. Berlin: Teubner, 243-249.

Wind, Edgar (2001): Das Experiment und die Metaphysik (1934). Hg. Bernhard Buschendorf. Frankfurt am Main: Suhrkamp. 
